\title{
(-)-Epigallocatechin-3-gallate reverses the expression of various tumor-suppressor genes by inhibiting DNA methyltransferases and histone deacetylases in human cervical cancer cells
}

\author{
MUNAWWAR ALI KHAN ${ }^{1}$, ARIF HUSSAIN ${ }^{2}$, MADHUMITHA KEDHARI SUNDARAM ${ }^{2}$, USAMA ALALAMI ${ }^{1}$, \\ DIAN GUNASEKERA ${ }^{2}$, LAVEENA RAMESH $^{2}$, AMINA HAMZA $^{2}$ and UZMA QURAISHI ${ }^{2}$ \\ ${ }^{1}$ Department of Natural Science and Public Health, College of Sustainability Sciences and Humanities, Zayed University, \\ Dubai; ${ }^{2}$ School of Life Sciences, Manipal University, Dubai, United Arab Emirates
}

Received July 15, 2014; Accepted January 29, 2015

DOI: $10.3892 /$ or.2015.3802

\begin{abstract}
There has been increasing evidence that numerous bioactive dietary agents can hamper the process of carcinogenesis by targeting epigenetic alterations including DNA methylation. This therapeutic approach is considered as a significant goal for cancer therapy due to the reversible nature of epigenetic-mediated gene silencing and warrants further attention. One such dietary agent, green tea catechin, (-)-epigallocatechin-3-gallate (EGCG) has been shown to modulate many cancer-related pathways. Thus, the present study was designed to investigate the role of EGCG as an epigenetic modifier in HeLa cells. DNA methyltransferase (DNMT) and histone deacetylase (HDAC) inhibition assays were conducted, and the transcription levels of DNMT3B and HDAC1 were assessed by enzymatic activity assay and RT-PCR, respectively. Furthermore, we studied the binding interaction of EGCG with DNMT3B and HDAC1 by molecular modeling as well as promoter DNA methylation and expression of retinoic acid receptor- $\beta(\operatorname{RAR} \beta)$, cadherin 1 (CDH1) and death-associated protein kinase-1 (DAPK1) in EGCG-treated HeLa cells by RT-PCR and MS-PCR. In the present study, time-dependent EGCG-treated HeLa cells were found to have a significant reduction in the enzymatic activity of DNMT and HDAC. However, the expression of DNMT3B was significantly decreased in a time-dependent manner whereas there was no significant change in HDAC1 expression. Molecular modeling data also supported the EGCG-mediated DNMT3B and HDAC1 activity inhibition. Furthermore, time-dependent exposure to EGCG resulted in reactivation of known tumor-suppressor genes (TSGs) in HeLa cells due to marked changes in the methylation of the
\end{abstract}

Correspondence to: Dr Arif Hussain, School of Life Sciences, Manipal University, P.O. Box 345050, Dubai, United Arab Emirates E-mail: dr.arifhussain@yahoo.co.in

Key words: EGCG, DNMT3B, HDAC1, epigenetic, reactivation, dietary agents promoter regions of these genes. Overall, the present study suggests that EGCG may have a significant impact on the development of novel epigenetic-based therapy.

\section{Introduction}

Many studies have proven that the process of carcinogenesis not only depends on genetic alterations but also on abnormal cellular memory, or epigenetic changes which are associated with a heritable gene expression profile critical for cancer development (1-4). An emerging perspective in therapeutic approaches for various types of cancers is to target epigenetic alterations due to their reversible nature, and these epigenetic alterations are manifested in both global changes in nucleosome packaging and in localized gene promoter changes of various tumor-suppressor genes (TSGs) which influence the transcription of genes during neoplastic initiation and progression $(1,2,4-7)$. Epigenetic alterations are mainly mediated by DNA methyltransferases (DNMTs), involved in DNA methylation, and histone deacetylases (HDACs), which play a pivotal role in histone deacetylation. However, there are several other classes of enzymes which are involved in post-translational modifications of histone tails and could be considered to represent an additional facet for the development of new anticancer therapies $(5,8,9)$.

Available epigenetic targeting modalities which are currently being investigated include HDAC and DNMT inhibitors. However, certain disadvantages that currently restrict the general use of these synthetic epigenetic drugs are that they exhibit a lack of specificity, have a short duration of action and may cause normal cells to change their functional and structural patterns with unforeseen effects (8-10). Burgeoning evidence in the last decade has provided unprecedented clues that diet and environmental factors directly influence epigenetic mechanisms in humans. Dietary polyphenols from green tea, turmeric, soybeans, broccoli and others have been shown to possess multiple cell regulatory activities within cancer cells. More recently, it has been noted that various dietary polyphenols may exert their chemopreventive effects in part by modulating various components of the epigenetic machinery, and therefore the drugs that target these alterations are being 
Table I. Residues defining the substrate binding pocket of HDAC1 and mDNMT3B.

Protein

Residues lining the substrate binding cavity

mDNMT3B

C-651, E-605, F-581, D-582, G-583, T-586, S-604, E-605, V-606, C-607, V-628,

G-648, S-649, P-650, C-651, N-652, S-655, V-657, 658, N-658, P-659, L-671, E-697,

V-699, V-700, A-701, R-731, A-732, R-733, R-773, I-774, K-777, S-778, N-779, S-780,

I-781, R-823, G-824, Q-827, K-828, G-831, R-832, S-833, W-834

HDAC1

H-140, H-141, D-176, H-178, D-264, L-271, F-109, W-135, A-136, G-137, L-139, G-149, C-151, F-205

Active site residues are underlined. HDAC, histone deacetylases.

studied in various human towards a better cancer treatment strategy $(11,12)$.

In green tea, among the most abundant chemical compounds, catechins which include (-)-epigallocatechin3-gallate (EGCG), have been shown to possess antioxidant, antiproliferative, anti-inflammatory, anti-angiogenic and antimetastatic activities, induce differentiation or apoptosis, arrest the cell cycle, inhibit telomerase activity and inhibit DNA adduct formation (13-18). Contemplating the potential role of EGCG as an epigenetic modifier, the present study was designed to investigate the inhibition of DNMTs and HDACs by EGCG and its effect on the expression of epigenetically modified TSGs including retinoic acid receptor- $\beta$ $(\mathrm{RAR} \beta)$, cadherin 1 (CDH1), death-associated protein kinase-1 (DAPK1) and MGMT in human cervical cancer cell line HeLa. To correlate the inhibition of DNMT and HDAC activity induced by EGCG, in silico molecular modeling and docking studies on DNMT3B and HDAC1 were performed.

\section{Materials and methods}

Cell culture. The human cervical carcinoma cell line HeLa was generously provided by Dr Tahir A Rizvi, UAE University, Al-Ain, United Arab Emirates (UAE). It was maintained in Dulbecco's modified Eagle's medium (DMEM) supplemented with $10 \%$ fetal bovine serum (FBS) and 100X Pen Strep (all from Sigma, USA) in a humidified atmosphere of $5 \% \mathrm{CO}_{2}$ in air at $37^{\circ} \mathrm{C}$.

Preparation of drugs. EGCG was obtained from Sigma. A stock solution of EGCG (10 mM) was prepared in water, sterile filtered with $0.2-\mu \mathrm{m}$ filters and stored at $-20^{\circ} \mathrm{C}$ in aliquots. Fresh EGCG solution was used in each experiment and further dilutions were made in complete medium to required concentrations of $25 \mu \mathrm{M}$ for the treatment of HeLa cells. A sub-stock of $500 \mu \mathrm{M}$ TSA was prepared from $5 \mathrm{mM}$ stock solution. A working concentration of $0.025 \mu \mathrm{M}$ was further used for the experiments. A stock solution of $219 \mathrm{mM}$ 5-aza-2'-deoxycytidine (5-Aza-dC) (Sigma) was prepared and further working concentration of $1 \mu \mathrm{M}$ was made from a $10 \mathrm{mM}$ sub-stock.

DNMT activity assay. HeLa cells were treated with EGCG $(25 \mu \mathrm{M})$ and 5-Aza-dC $(1 \mu \mathrm{M})$ for 3 days. After the treatment at various time points, the cells were harvested and nuclear extracts were prepared using the EpiQuik ${ }^{\mathrm{TM}}$ nuclear extraction kit (Epigentek, USA) as per the manufacturer's protocol.
Furthermore, the DNMT activity was assayed using the EpiQuik $^{\text {TM }}$ DNMT activity assay kit (Epigentek) as per the protocol instructions.

HDAC activity assay. The effect of EGCG on HDAC activity in the HeLa cells was determined using the EpiQuik ${ }^{\mathrm{TM}} \mathrm{HDAC}$ activity assay kit (Epigentek). Briefly, HeLa cells were treated with $25 \mu \mathrm{M}$ EGCG and $0.025 \mu \mathrm{M}$ TSA for 3 days, harvested and nuclear extracts were then prepared using the EpiQuik ${ }^{\mathrm{TM}}$ nuclear extraction kit following the manufacturer's instructions. Furthermore, DNMT activity was assayed using the EpiQuik ${ }^{\mathrm{TM}}$ DNMT activity assay kit as per protocol instructions.

Molecular modeling studies of DNMT3B and HDAC1 with $E G C G$. To address the interaction of EGCG with the epigenetic modulator enzymes HDAC1 and DNMT3B, we required validated $3 \mathrm{D}$ structures of the proteins. These structures were prepared and validated as detailed in our previous study [HDAC1 PDB ID: 4BKX (19); mDNMT3B was modeled from the structure of DNMT3A PDB ID: 2QRV (20)]. The substrate binding pocket was defined using the CASTp server as detailed in our previous study (unpublished data). The residues that constitute the substrate binding cavity of both proteins are listed in Table I.

The substrate binding pocket of mDNMT3B is fairly large and houses both the active residue (Cys-651) and the co-factor binding site (Glu-605). In several studies, the active site of class I HDACs has been paralleled to a tunnel that ends in a catalytically vital zinc ion (21). The cavity predicted by CASTp was similar to this description and includes the catalytic residue His-141 and $\mathrm{Zn}$ ion. Binding of EGCG in the same cavity where inhibitors 5-Aza-dC/TSA bind suggests that EGCG produces its inhibitory effects by a similar mechanism.

Docking. 3 Dimensional structures of EGCG, 5-Aza-dC and TSA in mol2 format were retrieved from the ZINC database (22). The SwissDock server, which uses EADock algorithm was used to perform blind docking of ligands (EGCG and 5-Aza-dC) with protein mDNMT3B and ligand (EGCG) with protein HDAC1 (23). All the residues of the proteins were held fixed and a binding pocket was not defined so as not to bias the docking towards the active site. The parameter selected for docking on the SwissDock server was 'accurate' with no flexibility of side chain of any amino acid of the target proteins. After calculating their energies using CHARMM, the different predicted binding modes of the ligand are ranked according to 
A

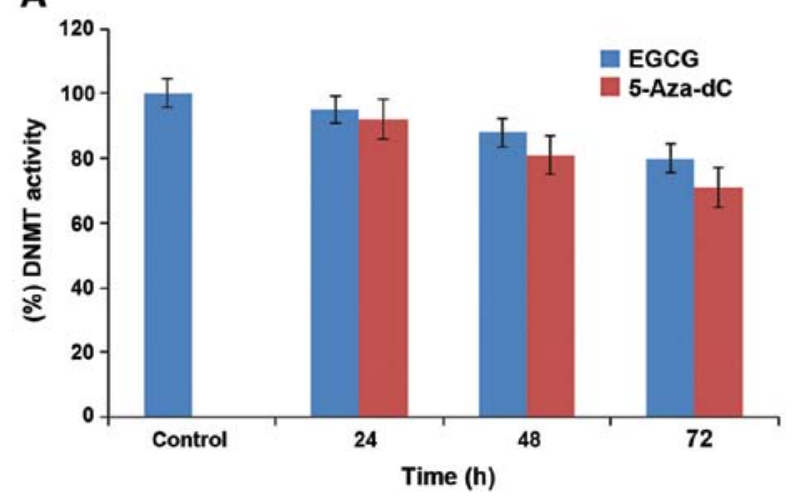

B

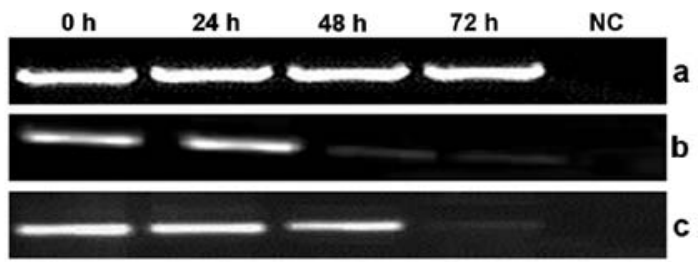

Figure 1. Effect of EGCG and 5-Aza-dC on DNA methyltransferases (DNMTs) in human cervical cancer HeLa cells. (A) HeLa cells treated with EGCG $(25 \mu \mathrm{M})$ and 5-Aza-Dc $(1.0 \mu \mathrm{M})$ exhibited significant inhibition in the activity of DNMTs in a time-dependent manner. Values are means \pm SD of 3 independent experiments. Each value for EGCG treatment differs from the control value $(\mathrm{P}<0.05)$. (B) HeLa cells treated with EGCG (25 $\mu \mathrm{M})$ and 5-Aza-Dc $(1.0 \mu \mathrm{M})$ exhibited a significant time-dependent reduction in the mRNA expression of DNMT3B in comparison to the untreated cells. Panel a, $\beta$-actin expression as an internal control; panel b, expression of DNMT3B following treatment with EGCG; and panel c, expression of DNMT3B following treatment with 5-Aza-dC. Lane 1, expression of DNMT3B gene in untreated HeLa cells; lanes 2-4, time-dependent alterations in the expression of DNMT3B upon treatment with EGCG and 5-Aza-Dc for 24, 48 and 72 h, respectively; lane 5, negative control (NC) for RT-PCR. EGCG, (-)-epigallocatechin-3-gallate; 5-Aza-dC, 5-aza2'-deoxycytidine.

their FullFitness scores. A more favorable binding mode is indicated by a more negative FullFitness score. UCSF-Chimaera, a molecular visualization software was used to perform the analyses of all docked poses (24).

Bisulfite modification and methylation-specific $P C R$ (MS-PCR). DNA was extracted from the EGCG-treated HeLa cells at various time points $(0,24,48$ and $72 \mathrm{~h})$ using the GenElute $^{\mathrm{TM}}$ Mammalian Genomic DNA Miniprep kit (Sigma) as per the manufacturer's instructions. After the DNA isolation, bisulphite modification and purification of modified DNA samples were carried out by the Imprint DNA Modification kit (Sigma) protocol. These modified DNA samples were used as a template for methylation-specific PCR (MSP), to distinguish between methylated and unmethylated promoter regions of the RAR $\beta, C D H 1$ and DAPK 1 genes using specific primer sets as previously described (methylated and unmethylated respectively) (25-27). MSP was performed on $50 \mathrm{ng}$ of bisulfite-treated DNA under the following conditions: initial denaturation at $95^{\circ} \mathrm{C}$ for $5 \mathrm{~min}$, followed by 35 amplification cycles (denaturation at $94^{\circ} \mathrm{C}$ for $30 \mathrm{sec}$, annealing $\mathrm{Tm}$ for $\operatorname{RAR} \beta, 56^{\circ} \mathrm{C}$; $\mathrm{CDH} 1,56^{\circ} \mathrm{C}$; DAPK $1,57^{\circ} \mathrm{C}$; $\mathrm{MGMT}, 55^{\circ} \mathrm{C}$; for $30 \mathrm{sec}$, and extension at $72^{\circ} \mathrm{C}$ for $45 \mathrm{sec}$ ) with a final extension at $72^{\circ} \mathrm{C}$ for $7 \mathrm{~min}$.

Reverse transcription-PCR. Total RNA isolation was carried out as per the manufacturer's protocol using the GenElute ${ }^{\mathrm{TM}}$ Mammalian Genomic Total RNA kit (Sigma) from $25 \mu \mathrm{M}$ EGCG-treated HeLa cells at various time points (24, 48 and $72 \mathrm{~h}$ ) including the untreated control. Reverse transcription of RNA to synthesize cDNA was performed using the ProtoScript M-MuLV Taq RT-PCR kit (New England Biolabs, USA) from $5 \mathrm{mg}$ of total RNA (at $42^{\circ} \mathrm{C}$ for $60 \mathrm{~min}$ ) followed by RT-PCR using gene-specific primers for $\beta$-actin, RAR $\beta$, CDH1, DAPK1, DNMTB and HDAC1. The PCR cycle was as follows: initial denaturation at $95^{\circ} \mathrm{C}$ for $5 \mathrm{~min}$, followed by 35 amplification cycles (denaturation at $94^{\circ} \mathrm{C}$ for $30 \mathrm{sec}$; annealing Tm for $\beta$-actin, $56^{\circ} \mathrm{C}$; $\operatorname{RAR} \beta, 56^{\circ} \mathrm{C}$; $\mathrm{CDH} 1,55.5^{\circ} \mathrm{C}$;
DAPK $1,56^{\circ} \mathrm{C}$; GSTP1, $55^{\circ} \mathrm{C}$; DNMTB, $56^{\circ} \mathrm{C}$; $\mathrm{HDAC} 1,56^{\circ} \mathrm{C}$ for $30 \mathrm{sec}$ and extension at $72^{\circ} \mathrm{C}$ for $45 \mathrm{sec}$ ), with a final extension at $72^{\circ} \mathrm{C}$ for $7 \mathrm{~min}$. The primer sequences used were described previously (28-32). Amplified products were visualized on a $2 \%$ agarose gel containing ethidium bromide.

\section{Results}

EGCG inhibits the activity of DNMTs and reduces the mRNA transcription level of DNMT3B in HeLa cells. EGCG treatment $(2.5 \mu \mathrm{M})$ at various time points $(24,48$ and $72 \mathrm{~h})$ was found to exert significant inhibitory action on the activity of DNMTs in HeLa cells treated in time-dependent manner and inhibited the enzyme activity by $5 \%(24 \mathrm{~h}), 12 \%$ (48 h) and $20 \%(72 \mathrm{~h})$ in HeLa cells compared with the untreated control (Fig. 1A). In contrast, time-dependent $(24,48$ and $72 \mathrm{~h})$ exposure of HeLa cells to $1 \mu \mathrm{M}$ 5-Aza-dC resulted in 8, 19 and $29 \%$ inhibition of DNMT activity in comparison to the untreated control. Furthermore, to decipher the probable reason for inhibition of enzyme activity, the findings were correlated with the changes in the expression of DNMT3B at the mRNA transcription level induced by EGCG in the HeLa cells. Untreated HeLa cells showed increased levels of DNMT3B mRNA whereas the cells treated with $25 \mu \mathrm{M}$ EGCG showed a significant decrease in the expression of DNMT3B in a time-dependent manner (24, 48 and 72 h) (Fig. 1B). In addition, an almost similar effect was observed in the 5-Aza-dC-treated HeLa cells and showed a time-dependent decrease in the expression of DNMT3B. $\beta$-actin was used as an internal control for sample comparison (Fig. 1B).

Effect of EGCG on HDAC activity and the expression of HDAC1 in HeLa cells. Chromatin modification is primarily regulated by HDAC enzymes and their high activity is linked with epigenetically silenced genes. The activity of HDACs in HeLa cells was determined by treating the cells with EGCG $(25 \mu \mathrm{M})$ for 24,48 and $72 \mathrm{~h}$, respectively. It was observed that HeLa cells treated with $25 \mu \mathrm{M}$ EGCG showed a time-dependent 


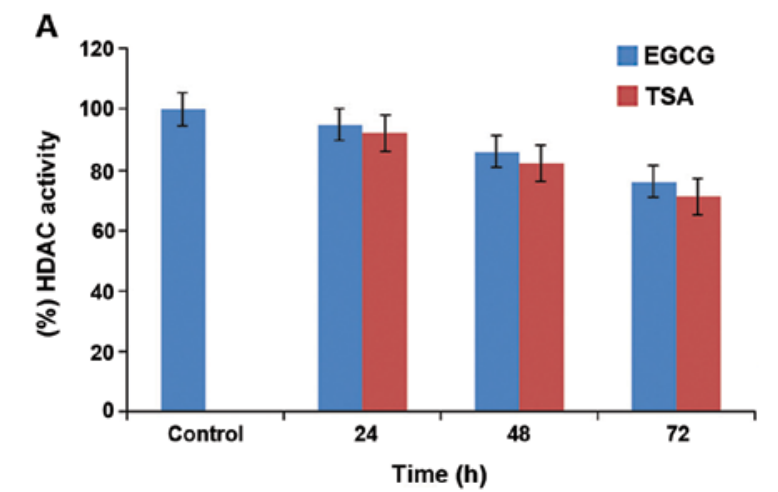

B

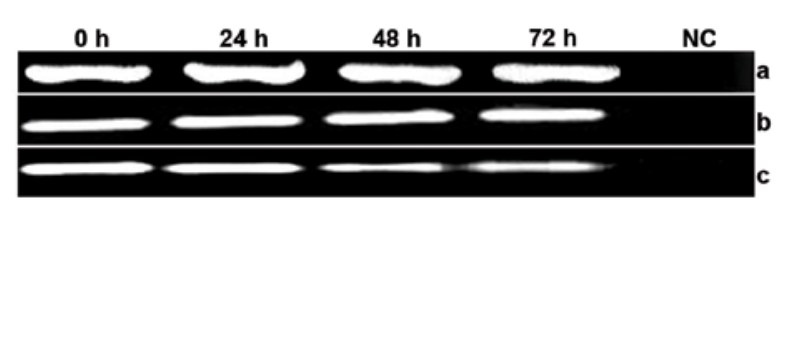

Figure 2. Effect of EGCG and TSA on histone deacetylases (HDACs) in human cervical cancer HeLa cells. (A) HeLa cells treated with EGCG (25 $\mu$ M) and TSA $(0.025 \mu \mathrm{M})$ exhibited significant inhibition in the activity of HDAC in a time-dependent manner. Values are means \pm SD of 3 independent experiments. Each value for EGCG treatment differs from the control value $(\mathrm{P}<0.05)$. (B) HeLa cells treated with EGCG $(25 \mu \mathrm{M})$ did not show any detectable changes in regards to the expression of HDAC1 whereas treatment with $0.025 \mu \mathrm{M}$ TSA decreased the expression of HDAC1 in the HeLa cells in a time-dependent manner in comparison to the untreated cells. Panel a, $\beta$-actin expression as an internal control; panel b, expression of HDAC1 following treatment with EGCG; and panel c, expression of HDAC1 following treatment with TSA. Lane 1, expression of HDAC gene in untreated HeLa cells; lanes 2-4, time-dependent modulation in the expression of HDAC1 upon treatment for 24, 48 and $72 \mathrm{~h}$, respectively; lane 5, negative control (NC) for RT-PCR. EGCG, (-)-epigallocatechin-3-gallate.

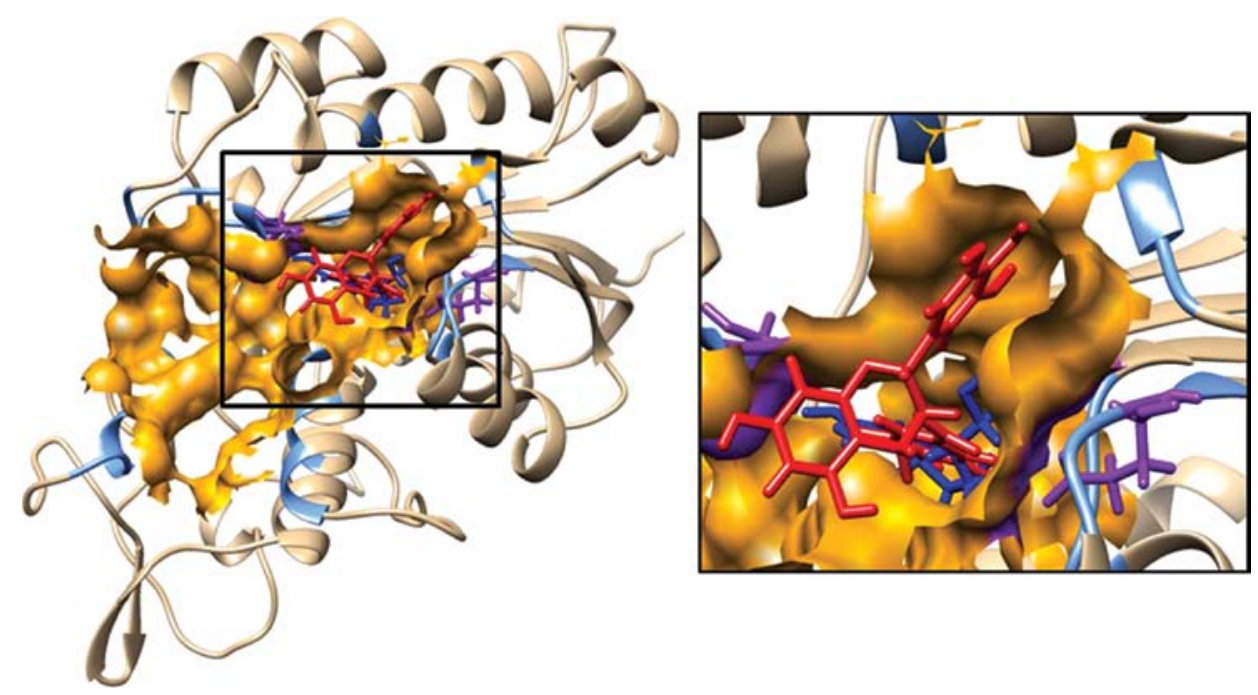

Figure 3. Predicted interaction between ligands (EGCG and 5-Aza-dC) with mDNMT3B. The mDNMT3B is depicted in a ribbon representation showing docking models of EGCG in red and 5-Aza-dC in blue and the residues defining the pocket as light blue. The inset focuses on the binding pocket shown in orange. Active site C-651 and cofactor binding E-605 are shown in purple solid bonds. EGCG, (-)-epigallocatechin-3-gallate; 5-Aza-dC, 5-aza-2'-deoxycytidine.

decrease of 5, 14 and 24\% in HDAC activity (Fig. 2A). It was also observed that exposure to the HDAC inhibitor $(0.025 \mu \mathrm{M}$ TSA) caused a time-dependent $(24,48$ and $72 \mathrm{~h})$ decrease in the activity of HDACs in the HeLa cells and caused 8, 18 and $29 \%$ inhibition, respectively (Fig. 2A). Furthermore, it was also examined whether HDAC activity may or may not be correlated with a decrease in HDAC1 expression. It was found that EGCG $(25 \mu \mathrm{M})$-treated HeLa cells did not show any significant changes in the expression of HDAC1 in comparison to the untreated cells (Fig. 2B). However, TSA-treated HeLa cells showed a significant reduction in the expression of HDAC1 at 24, 48 and 72 h, respectively (Fig. 2B).

EGCG interacts with DNMT3B and HDAC1: An in silico molecular model. In order to investigate the possible mechanism by which EGCG inhibits HDAC1 and DNMT3B, an in silico theoretical molecular modeling approach was used. The cavity predicted by CASTp which included the HDAC1 residues expected to interact with ligand TSA as well as the active site His-141 and $\mathrm{Zn}$ ion was taken to be the substrate binding site of HDAC1. The CASTp predicted cavity that included the active site residue Cys-651 and co-factor binding residue Glu-605 was defined as the substrate-binding cavity for DNMT3B. Previously, we evaluated the structure and the predicted cavity of DNMT3B by docking its well-known inhibitor 5-Aza-dC, using the SwissDock server. The same methodology was used to dock EGCG on HDAC1 and mDNMT3B. FullFitness and Gibbs free energy $(\Delta G)$ of each run (256 runs) of the docking experiment were evaluated. Favorable binding modes were scored based on FullFitness and cluster formation. The value of FullFitness was used to rank clusters for further analysis.

Interaction of EGCG with mDNMT3B. The docking of 5-Aza-dC, a well-known inhibitor of DNMT3B, was performed first to define the substrate binding site of the 
Table II. Docking results of ligands (EGCG, TSA and 5-Aza-dC) on receptors (HDAC1 and mDNMT3B).

\begin{tabular}{|c|c|c|c|c|c|c|}
\hline Receptor & Ligand & Clusters & Cluster ranks & $\begin{array}{c}\text { Total } \\
\text { elements }\end{array}$ & $\begin{array}{l}\text { FullFitness } \\
(\mathrm{kcal} / \mathrm{mol})\end{array}$ & $\begin{array}{l}\text { Estimated } \Delta \mathrm{G} \\
\quad(\mathrm{kcal} / \mathrm{mol})\end{array}$ \\
\hline mDNMT3B & 5-Aza-dC & $15 / 43$ & $0-1,3-5,7,17,19,24,34,36-37,39,42,43$ & $73 / 256$ & -2071.4 & -9.5 \\
\hline mDNMT3B & EGCG & $21 / 44$ & $\begin{array}{l}8,10,11,14-16,18,19,24-26,29-30,35-37 \\
39,40,41,43,44\end{array}$ & $120 / 256$ & -1866.53 & -6.94 \\
\hline HDAC1 & EGCG & $3 / 31$ & $0,2,9$ & $23 / 256$ & -2042.02 & -7.48 \\
\hline
\end{tabular}

Column 3 represents clusters within substrate binding cavity/total clusters. EGCG, (-)-epigallocatechin-3-gallate; 5-Aza-dC, 5-aza-2'-deoxycytidine; HDAC, histone deacetylases; $\Delta \mathrm{G}$, Gibbs free energy.

Table III. Residues of HDAC1 and mDNMT3B within 5 A of EGCG.

\begin{tabular}{ll}
\hline Protein & \multicolumn{1}{c}{ Residues within 5 Å of EGCG } \\
\hline mDNMT3B & C-651, E-605, V-606, C-607 F-581, D-582, G-583, S-610, D-627, V-628, R-629, G-648, S-649, P-650, \\
& N-652, T-668, L-671, K-828, R-832, S-833 \\
HDAC1 & $\underline{\text { H-141, G-27, H-28, P-29, D-99, G-149, S-148, F-150, H-178, E-203, Y-204, F-205, R-270, L271, Y-303 }}$
\end{tabular}

Catalytically active residues are underlined. HDAC, histone deacetylases; EGCG, (-)-epigallocatechin-3-gallate; 5-Aza-dC, 5-aza-2'-deoxycytidine.

protein. The docking results produced 43 clusters of ligand around the modeled catalytic domain of DNMT3B. Fifteen clusters were found to bind in the CASTp predicted cavity of which the ligand models in the top 2 clusters $(0$ and 1$)$ were found to be in close proximity to the active site Cys-651 and cofactor (S-adenosyl methionine) binding residue Glu-605. The distance of the closest atoms of Cys-651 and Glu-605 from the most favorable docking model of 5-Aza-dC was found to be 1.86 and $2.34 \AA$, respectively. The docking result of 5-Aza-dC on mDNMT3B established the validity of the substrate binding cavity predicted by CASTp. Following this, the binding of the ligand EGCG on mDNMT3B was probed by performing their docking using the SwissDock server. The docking results produced 34 clusters of ligand EGCG around the modeled catalytic domain of DNMT3B. When the clusters were analyzed it was found that 15 of them could bind in the substrate binding cavity constituting a total of 120 predicted binding elements out of a total of 256. It is striking that almost half of the total predicted elements are docked in the substrate binding cavity. The predicted clusters include top ranked cluster 1 with 16 elements as well as several other clusters. While cluster rank 0 is energetically lower it was not considered as the predicted binding of EGCG was on the outer edge of the catalytic domain, a region which may not represent a pocket or be accessible to the ligand in the complete protein. Notably, it was observed that the gallate moiety of EGCG was consistently positioned in close proximity to the important residues in the substrate binding cavity. This may be interpreted as an indication of its important role in the binding of EGCG to the protein. The amino acids, Arg 832, Arg 823, Ser 778 and Asn 652, were repeatedly observed to be involved in hydrogen bond formation with EGCG. Table II shows the summary result of SwissDock docking and the FullFittness and estimated $\Delta \mathrm{G}$ values for the most favorable interaction. Observation of the majority of clusters, including the top ranked ones, in the cavity strongly suggests that the preferred binding of EGCG on mDNMT3B is within the substrate binding cavity. Fig. 3 shows a representative image of the binding of EGCG and 5-Aza-dC on the protein mDNMT3B. Table III lists all of the mDNMT3B residues within $5 \AA$ of the most energetically favorable docking model of EGCG.

Interaction of EGCG with HDAC1. The docking results produced 31 clusters of ligand EGCG around the complete protein HDAC1. Analysis of these clusters showed that 3 of these clusters were able to bind in the substrate binding cavity. These clusters together contained a total of 23 elements out of 256 predicted binding modes. Notably, these clusters included the top ranked clusters 0 and 2 in addition to cluster 9. Table II shows the SwissDock docking results as well as the FullFittness and estimated $\Delta \mathrm{G}$ values for the most favorable interaction. The lowest energy model of cluster rank zero was considered to be the most favorable interaction. The presence of two high ranking clusters strongly suggests that the preferred binding of EGCG on HDAC1 is within the substrate binding cavity. The gallate moiety of EGCG seems to play an important role as it is oriented towards the active residue in the least energy model. Fig. 4 shows the most energetically favorable binding of EGCG on HDAC1 as well as TSA on HDAC1 which was achieved by superimposing the crystal structure of HDAC8 bound to TSA on HDAC1. The image visibly records that the predicted binding orientation of EGCG and transposed TSA overlap the same region on HDACl. It is noteworthy that the key catalytic residues such as the active site His-141 and Zn ion are within $5 \AA$ of the ligands. Table III lists all the HDAC1 residues within $5 \AA$ of the most energetically favorable docking model of EGCG. 


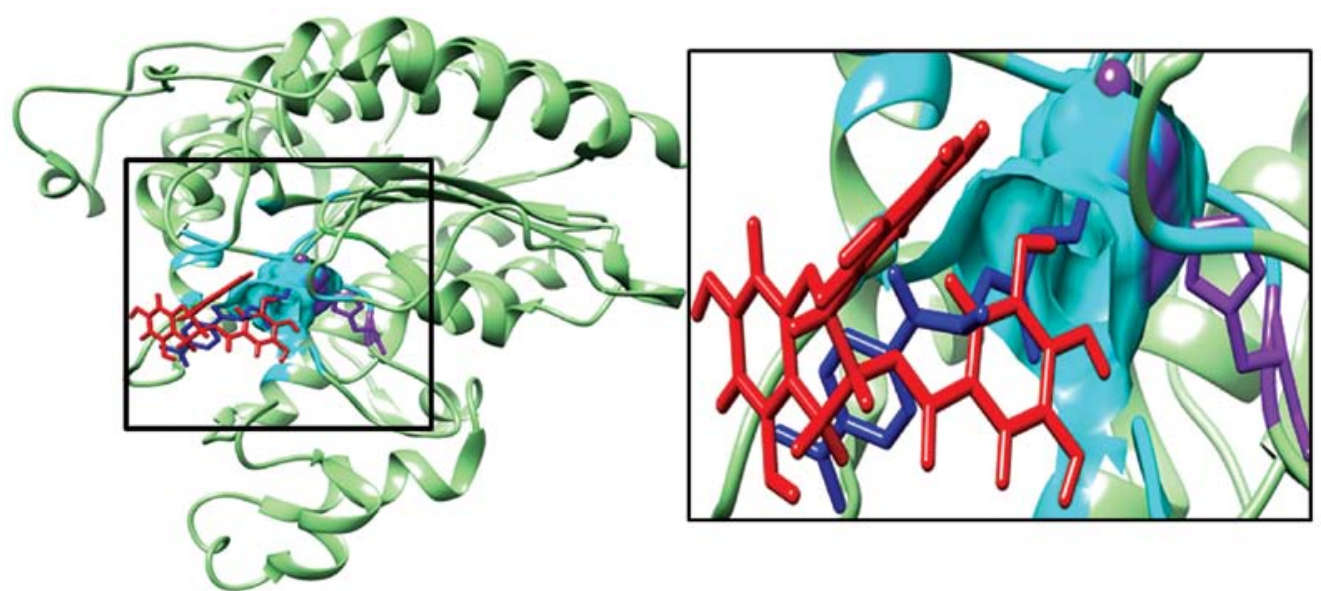

Figure 4. Predicted interaction between ligands (EGCG and TSA) with HDAC1. The HDAC1 protein is depicted in a ribbon representation showing the docking model of EGCG in red and TSA in blue and the residues defining the pocket as light blue. The TSA structure was transformed from HDAC 8 by superimposition on HDAC1. The inset focuses on the binding pocket shown in orange. The active site H-141 and Zn ion are highlighted in purple. EGCG, (-)-epigallocatechin-3-gallate; HDAC, histone deacetylases.

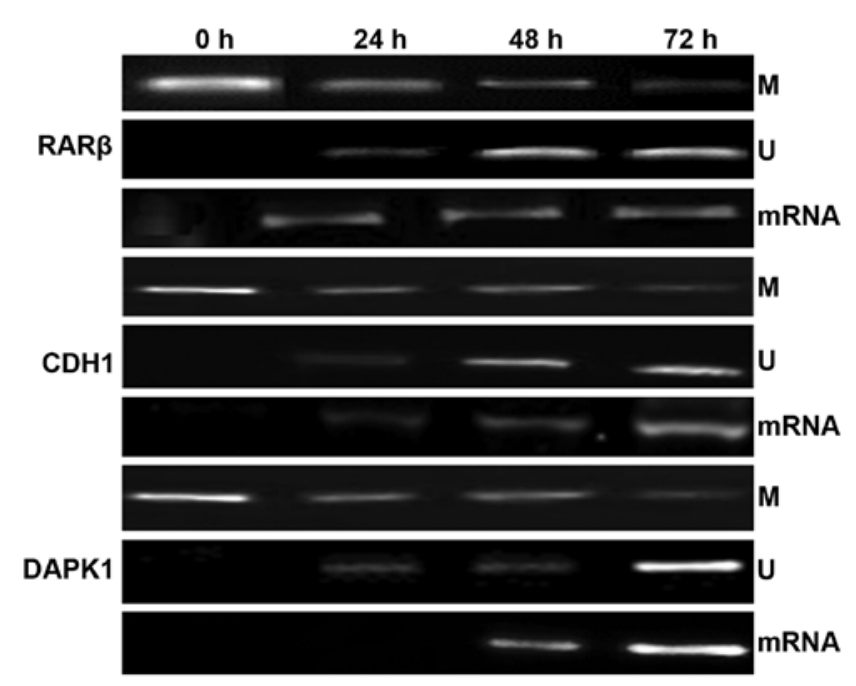

Figure 5. Alterations in the methylation status and mRNA expression levels of RAR $\beta$, CDH1 and DAPK1 genes after treatment with EGCG. Panels M and U show the methylation-specific bands (M) and unmethylation-specific bands (U): lane 1, methylation status of these genes in untreated HeLa cells; lane 2-4, timedependent modulation in the methylation status of RAR $\beta, C D H 1$ and DAPK1 genes for 24, 48 and $72 \mathrm{~h}$, respectively. Panel mRNA shows the expression of these genes after treatment with EGCG: lane 2-4, time-dependent modulation in the expression of HDAC1 upon treatment for 24, 48 and $72 \mathrm{~h}$, respectively, whereas lane 1 shows the expression of these genes in untreated HeLa cells.

EGCG reactivates $R A R \beta, C D H 1$ and DAPK1 genes through reversal of hypermethylation in HeLa cells. Many TSGs are inactivated via aberrant epigenetic changes at their promoter region during cancer development, and due to the reversible nature of these changes epigenetic-based cancer treatment strategy is attracting more research interest. To determine whether EGCG-induced inhibition of DNMT3B and HDAC1 results in epigenetic changes leading to reactivation of various TSGs such as RAR $\beta$, CDH1 and DAPK1, expression of these genes was further correlated with the changes in the methylation of their promoter regions. It was observed that HeLa cells treated with $25 \mu \mathrm{M}$ EGCG exhibited a time-dependent (24, 48 and 72 h) significant increase in the expression of RAR $\beta$,
CDH1 and DAPK1 at the mRNA level in comparison to the untreated cells (Fig. 5). Furthermore, it was observed that the changes in the expression of these genes were associated with the reversal of 5' $\mathrm{CpG}$ island methylation of their promoter regions. Treated HeLa cells were subjected to MSP with methylation-specific and unmethylation-specific sets of primers. EGCG treated HeLa cells showing amplification only after using methylated and unmethylated primer set, were considered as hypermethylated and unmethylated respectively. From the MSP results, it was observed that RAR $\beta, \mathrm{CDH} 1$ and DAPK1 genes were found to be hypermethylated in the untreated HeLa cells. However, after treatment with EGCG, the 5' $\mathrm{CpG}$ island methylation of the promoters of the these genes was reversed as evident by the decreased level of amplimer intensity with methylation-specific primers whereas it was significantly increased with the unmethylated set of primers in a time-dependent manner (Fig. 5B). These results confirm that EGCG restores the expression of RAR $\beta$, CDH1 and DAPK1 genes via the reversal of 5' CpG island methylation and inhibition of epigenetic modulators such as DNMTs and HDACs in HeLa cells.

\section{Discussion}

In recent years, researchers in the field of epigenetics have made great strides in understanding the many molecular sequences and patterns that determine which genes can be turned on and off. The present study has made it increasingly clear that in addition to genetic changes, epigenetic alterations are also very critical for cancer development $(1,2)$. Unlike genetic alterations, certain epigenetic alterations i.e. aberrant DNA methylation and histone acetylation which lead to gene inactivation of various tumor-suppressor genes (TSGs) in cancer cells, can be reversed using epigenetic drugs, making them valuable therapeutic targets for cancer therapy. The drugs that target these epigenetic alterations are termed epigenetic drugs. Currently, two classes of synthetic epigenetic drugs based on nucleoside and non-nucleoside nature are being investigated as DNA methyltransferase (DNMT) and histone deacetylase 
(HDAC) inhibitors $(33,34)$. However, there are many drawbacks of these drugs such as lack of specificity, short duration of action and cytotoxicity towards normal cells that currently restrict the general use of these drugs (8-10).

More importantly, a wide gamut of reports indicates that dietary phytochemicals can modulate epigenetic alterations and alter susceptibility to various diseases including cancer. In the development of epigenetic drugs as inhibitors of DNMTs and HDACs, dietary phytochemicals are gaining more interest due to their safe therapeutic profile $(11,12,35)$. One such promising agent is (-)-epigallocatechin-3-gallate (EGCG). Its anticarcinogenic effects via various molecular pathways have been established in previous studies (13-18). Previous studies in our laboratory also found that EGCG was selectively cytotoxic towards cancer cells and inhibited the growth of cancer cells in a dose- and time-dependent manner and the $\mathrm{EC}_{50}$ was found to be $100 \mu \mathrm{M}$ for a duration of $24 \mathrm{~h}$ and $50 \mu \mathrm{M}$ for $48 \mathrm{~h}$ (13). From these results, we selected a sublethal dose (25 $\mu$ M EGCG) for the present study.

DNA methylation and histone acetylation and de-acetylation are most commonly occurring epigenetic events taking place in the mammalian genome and control the expression of various genes including TSGs (36-38). A critical step in DNA methylation involves DNMTs, the enzymes that catalyze the methylation process. It has been established that inactivation of many TSGs via DNA methylation and histone modification is due to the high enzymatic activity of DNMTs and HDACs. Various studies have indicated the importance of DNMTs and HDACs as epigenetic targets leading to the development of many synthetic drugs that function as their inhibitors (7,39-41). During our screening study to determine whether EGCG affects epigenetic modulation via DNMT and HDAC enzymes, it was demonstrated that EGCG-treated HeLa cells showed significant inhibition in the enzymatic activity of these enzymes in a time-dependent manner (Figs. 1A and 2A). Almost similar effects were observed in HeLa cells after treatment with their respective well characterized inhibitors (Figs. 1A and 2A). These results are consistent with other studies in which many dietary agents along with EGCG have shown their inhibitory action on the enzymatic activity of DNMT and HDAC enzymes.

Several scientific studies have demonstrated that DNMT3B, one of the important enzymes of the DNMT family and HDAC1, one of the members of the HDAC enzyme family, are overexpressed in various types of cancers (42-48). To determine whether EGCG has any effect on the mRNA transcription level of these enzymes, the mRNA expression level was studied in EGCG-treated HeLa cells. It was found that treated Hela cells showed a time-dependent decrease in the expression of DNMT3B whereas no significant changes in the expression of HDACl in the EGCG-treated HeLa cells were observed (Figs. 1B and 2B). However, time-dependent exposure of 5-Aza-dC and TSA decreased the expression of DNMT3B and HDAC1, respectively, in the HeLa cells. The present study is in line with various studies in which various dietary agents act as epigenetic modifiers and modulate the epigenetic changes via targeting DNMTs and HDACs (49-52).

Furthermore, in silico molecular modeling studies were performed to ascertain whether the direct interaction of EGCG with modeled DNMT3B and HDAC1 explains the EGCG- mediated enzymatic inhibition of DNMTs and HDACs. Our analyses of the predicted docking results emphatically indicate that EGCG directly binds in the substrate binding pocket of these two enzymes.

The substrate binding pocket of DNMT3B was delineated using computational and knowledge-based approaches. Docking of 5-Aza-dC in concurrence with knowledge concerning the active sites and cofactor binding sites helped us to identify with certainty the substrate binding pocket for DNMT3B. Table III lists the residues lining the pocket including active site C-651 and E-605 essential for binding to SAM, which is responsible for methyltransferase activity. Fig. 4 illustrates the docking of EGCG and 5-Aza-dC on mDNMT3B. Notably, a very high proportion (15 out of 34 , containing 120 out of 256 independent docking runs) of predicted top ranked clusters for EGCG was observed in the defined substrate binding pocket of mDNMT3B. These results show that EGCG and 5-Aza-dC dock within the substrate binding cavity of the protein and therefore may have a similar mechanism of protein inhibition by preventing the entry of the natural ligand into the active site. Remarkably, we observed that the gallate moiety frequently binds in close proximity to the active residue or the other key residues such as Arg 832, Arg 823, Ser 778 and Asn 652. Docking studies on DNMT1 have proposed that the D ring/gallate moiety of EGCG occupies a binding pocket analogous to the pyrimidyl ring of cytosine and could be significant for its direct inhibition of the enzyme $(53,54)$. The recurrent involvement of the amino acids Arg 832, Arg 823, Ser 778 and Asn 652, in hydrogen bond formation with EGCG underscores their importance in the catalytic pocket. Further emphasis supporting this hypothesis is lent by a report on the inhibition of DNMT3B by nanomycin following key interactions with Arg 731, Arg 733, Arg 832 and Asn $652(55,56)$. These residues have been reported to be involved in the DNA methylation mechanism (56). Our docking study also reinforces the role of this residue. Hence, we can expect that the residue Arg 832 is important for inhibitor binding and stability and when involved in binding to the inhibitor will result in enzyme inactivity. In Table III we list those residues of DNMT3B which may be involved in interactions with EGCG.

In Fig. 3, the best energetically favored model of EGCG docking on HDACl overlaps with the binding site of TSA. As a result of the binding of EGCG to HDAC1, we can expect that it would impede the entry and catalysis of the actual substrate. This leads us to propose that EGCG may be functionally similar to TSA, as an HDAC inhibitor. The residues of HDAC1 that interact with the most energetically favorable docking model of EGCG are listed in Table III.

These indications are strong enough for us to recommend that given the better safety profile of EGCG in comparison to 5-Aza-dC and TSA, EGCG is a better candidate as a similarly functioning epigenetic modulator. Our molecular modeling and docking studies not only successfully explain the mechanism of action of EGCG in inhibiting epigenetic modulating enzymes but also paves the way to embark upon further possibilities such as structure-guided optimization studies and pharmacophore modeling.

Many reports have shown that inactivation of tumorsuppressor genes is associated with promoter hypermethylation 
in various types of cancers (57-59). It is now well established that methylation of DNA and modifications of histones are intimately interconnected. For example, DNMTs can bind to HDACs, thereby repressing gene transcription through histone deacetylase activity. There is a family of proteins collectively referred to as methyl $\mathrm{CpG}$ binding proteins (MBDs) that share a methyl-CpG binding domain. These proteins are thought to recruit HDACs to methyl-CpG-enriched regions of the genome to repress transcription (4-7). In the present study, the possible epigenetic modulatory effects of EGCG on various epigenetically silenced TSGs including RAR $\beta$, CDH1 and DAPK1 in HeLa cells were studied and were also correlated with its ability to inhibit DNMTB and HDAC1 activity. Possibly, EGCG-mediated inhibition of DNMTs and HDACs favors to change the DNA methylation and histone de-acetylation status which results in the reactivation of these genes. Extensive studies have found that the RAR $\beta, \mathrm{CDH} 1$ and DAPK1 genes are transcriptionally silenced due to promoter hypermethylation during the development of various human cancers (60-64). In the present study, it was observed that the RAR $\beta, C D H 1$ and DAPK1 genes were hypermethylated and this was correlated with their respective expression which was found to be undetectable in the untreated HeLa cells (Fig. 5). However, after treatment with EGCG, HeLa cells showed timedependent changes in the methylation status as the bands in the methylated panel were reduced and significantly detectable in the unmethylated panel which was further linked with restoration of the expression of RAR $\beta, \mathrm{CDH} 1$ and DAPK 1 genes during the time exposure (Fig. 5). The present study is similar to several other studies which have shown that various dietary agents including EGCG reactivate many TSGs via modulation of various epigenetic pathways $(49,51,52,59,65,66)$.

The present study demonstrated that EGCG functions as a potential epigenetic modifier and acts via inhibitory action on the activity of DNMTs and HDACs and reactivates epigenetically silenced TSGs by altering the methylation status of the promoters of these genes. EGCG can be used as an effective inhibitor of DNMTs and HDACs to prevent cancer. However, further mechanistic studies as well as in vivo studies and clinical trials are needed to determine the efficacy of EGCG for therapeutic purposes as an epigenetic drug.

\section{Acknowledgements}

The authors are grateful to Dr Kota Reddy, Academic President and Dr Firdos Alam Khan, Chairperson, School of Life Sciences, Manipal University, Dubai, for their constant support and encouragement. The present study was financially supported by Zayed University Research Incentive Fund (RIF) (activity code: R13052) and the Intramural Research Program at Manipal University, Dubai, United Arab Emirates (UAE).

\section{References}

1. Ting AH, McGarvey KM and Baylin SB: The cancer epigenome components and functional correlates. Genes Dev 20: 3215-3231, 2006.

2. Choi JD and Lee JS: Interplay between epigenetics and genetics in cancer. Genomics Inf 11: 164-173, 2013.

3. Flis S, Gnyszka A and Flis K: DNA methyltransferase inhibitors improve the effect of chemotherapeutic agents in SW48 and HT-29 colorectal cancer cells. PLoS One 27: e92305, 2014.
4. Geurts van Kessel A: The cancer genome: from structure to function. Cell Oncol 37: 155-165, 2014.

5. Tallen $\mathrm{G}$ and Riabowol K: Keep-ING balance: Tumor suppression by epigenetic regulation. FEBS Lett 588: 2728-2742, 2014.

6. Wee S, Dhanak D, Li H, Armstrong SA, Copeland RA, Sims R, Baylin SB, Liu XS and Schweizer L: Targeting epigenetic regulators for cancer therapy. Ann NY Acad Sci 1309: 30-36, 2014.

7. Jeong HM, Kwon MJ and Shin YK: Overexpression of cancerassociated genes via epigenetic derepression mechanisms in gynecologic cancer. Front Oncol 4: 12, 2014.

8. Subramaniam D, Thombre R, Dhar A and Anant S: DNA methyltransferases: A novel target for prevention and therapy. Front Oncol 4: 80, 2014.

9. Campbell RM and Tummino PJ: Cancer epigenetics drug discovery and development: The challenge of hitting the mark. J Clin Invest 124: 64-69, 2014.

10. Cantley MD and Haynes DR: Epigenetic regulation of inflammation: Progressing from broad acting histone deacetylase (HDAC) inhibitors to targeting specific HDACs. Inflammopharmacology 21: 301-307, 2013.

11. Henning SM, Wang P, Carpenter CL and Heber D: Epigenetic effects of green tea polyphenols in cancer. Epigenomics 5: 729-741, 2013.

12. Yang P, He X and Malhotra A: Epigenetic targets of polyphenols in cancer. J Environ Pathol Toxicol Oncol 33: 159-165, 2014.

13. Sharma C, Nusri Q-A, Begum S, Javed E, Rizvi TA and Hussain A: (-)-Epigallocatechin-3-gallate induces apoptosis and inhibits invasion and migration of human cervical cancer cells. Asian Pac J Cancer Prev 13: 4815-4822, 2012.

14. Lin CH, Chao LK, Hung PH and Chen YJ: EGCG inhibits the growth and tumorigenicity of nasopharyngeal tumor-initiating cells through attenuation of STAT3 activation. Int J Clin Exp Pathol 7: 2372-2381, 2014.

15. Siddiqui IA, Bharali DJ, Nihal M, Adhami VM, Khan N, Chamcheu JC, Khan MI, Shabana S, Mousa SA and Mukhtar H: Excellent anti-proliferative and pro-apoptotic effects of (-)-epigallocatechin-3-gallate encapsulated in chitosan nanoparticles on human melanoma cell growth both in vitro and in vivo. Nanomedicine 10: 1619-1626, 2014.

16. Luo HQ, Xu M, Zhong WT, Cui ZY, Liu FM, Zhou KY and Li XY: EGCG decreases the expression of HIF-1 $\alpha$ and VEGF and cell growth in MCF-7 breast cancer cells. J BUON 19: 435-439, 2014.

17. Butt MS, Ahmad RS, Sultan MT, Nasir Qayyum MM and Naz A: Green tea and anticancer perspectives: Updates from last decade. Crit Rev Food Sci Nutr 55: 792-805, 2015.

18. Yokoyama M, Noguchi M, Nakao Y, Ysunaga M, Yamasaki F and Iwasaka T: Antiproliferative effects of the major tea polyphenol, (-)-epigallocatechin gallate and retinoic acid in cervical adenocarcinoma. Gynecol Oncol 108: 326-331, 2008.

19. Millard CJ, Watson PJ, Celardo I, Gordiyenko Y, Cowley SM, Robinson CV, Fairall L and Schwabe JW: Class I HDACs share a common mechanism of regulation by inositol phosphates. Mol Cell 51: 57-67, 2013.

20. Jia D, Jurkowska RZ, Zhang X, Jeltsch A and Cheng X: Structure of Dnmt3a bound to Dnmt3L suggests a model for de novo DNA methylation. Nature 449: 248-251, 2007.

21. Thangapandian S, John S, Lee Y, Arulalapperumal V and Lee KW: Molecular modeling study on tunnel behavior in different histone deacetylase isoforms. PLoS One 7: e49327, 2012.

22. Irwin JJ, Sterling T, Mysinger MM, Bolstad ES and Coleman RG: ZINC: A free tool to discover chemistry for biology. J Chem Inf Model 52: 1757-1768, 2012.

23. Grosdidier A, Zoete V and Michielin O: SwissDock, a proteinsmall molecule docking web service based on EADock DSS Nucleic Acids Res 39: W270-W277, 2011.

24. Pettersen EF, Goddard TD, Huang CC, Couch GS, Greenblatt DM, Meng EC and Ferrin TE: UCSF Chimera - a visualization system for exploratory research and analysis. J Comput Chem 25: 1605-1612, 2004.

25. El-Shakankiry NH and Mossallam GI: p15 (INK4B) and E-cadherin CpG island methylation is frequent in Egyptian acute myeloid leukemia. J Egypt Natl Canc Inst 18: 227-232, 2006.

26. Suzuki M, Shigematsu H, Iizasa T, Hiroshima K, Nakatani Y, Minna JD, Gazdar AF and Fujisawa T: Exclusive mutation in epidermal growth factor receptor gene, HER-2, and KRAS, and synchronous methylation of nonsmall cell lung cancer. Cancer 106: 2200-2207, 2006. 
27. Yaqinuddin A, Qureshi SA, Qazi R and Abbas F: Downregulation of DNMT3b in PC3 cells effects locus-specific DNA methylation, and represses cellular growth and migration. Cancer Cell Int 8: 13, 2008.

28. Saito Y, Kanai Y, Sakamoto M, Saito H, Ishii H and Hirohashi S: Overexpression of a splice variant of DNA methyltransferase $3 b$, DNMT3b4, associated with DNA hypomethylation on pericentromeric satellite regions during human hepatocarcinogenesis. Proc Natl Acad Sci USA 99: 10060-10065, 2002.

29. Goelden U, Ukena SN, Pfoertner S, Hofmann R, Buer J and Schrader AJ: RAR-beta(1) overexpression in chromophobe renal cell carcinoma: A novel target for therapeutic intervention? Exp Oncol 27: 220-224, 2005.

30. Wang AG, Kim SU, Lee SH, Kim SK, Seo SB, Yu DY and Lee DS: Histone deacetylase 1 contributes to cell cycle and apoptosis. Biol Pharm Bull 28: 1966-1970, 2005.

31. Jin Y, Blue EK and Gallagher PJ: Control of death-associated protein kinase (DAPK) activity by phosphorylation and proteasomal degradation. J Biol Chem 281: 39033-39040, 2006.

32. Chang J, Nicolau MM, Cox TR, Wetterskog D, Martens JW, Barker HE and Erler JT: LOXL2 induces aberrant acinar morphogenesis via ErbB2 signaling. Breast Cancer Res 15: R67, 2013.

33. Capobianco E, Mora A, La Sala D, Roberti A, Zaki N, Badidi E, Taranta $\mathrm{M}$ and Cinti C: Separate and combined effects of DNMT and HDAC inhibitors in treating human multi-drug resistant osteosarcoma HosDXR150 cell line. PLoS One 9: e95596, 2014.

34. Al-Rayyan N, Litchfield LM, Ivanova MM, Radde BN, Cheng A, Elbedewy A and Klinge CM: 5-Aza-2-deoxycytidine and trichostatin A increase COUP-TFII expression in antiestrogen-resistant breast cancer cell lines. Cancer Lett 347: 139-150, 2014.

35. Thakur VS, Deb G, Babcook MA and Gupta S: Plant phytochemicals as epigenetic modulators: Role in cancer chemoprevention. AAPS J 16: 151-163, 2014.

36. Jiao F, Wang X, Yan Z, Liu C, Yue Z, Li Z, Ma Y, Li Y and Wang J: Effect of dynamic DNA methylation and histone acetylation on $c P o u V$ expression in differentiation of chick embryonic germ cells. Stem Cells Dev 22: 2725-2735, 2013.

37. Herranz M and Esteller M: DNA methylation and histone modifications in patients with cancer: Potential prognostic and therapeutic targets. Methods Mol Biol 361: 25-62, 2007.

38. Oyer JA, Chu A, Brar S and Turker MS: Aberrant epigenetic silencing is triggered by a transient reduction in gene expression. PLoS One 4: e4832, 2009.

39. Zopf S, Ocker M, Neureiter D, Alinger B, Gahr S, Neurath MF and Di Fazio P: Inhibition of DNA methyltransferase activity and expression by treatment with the pan-deacetylase inhibitor panobinostat in hepatocellular carcinoma cell lines. BMC Cancer 12: 386, 2012.

40. Zhu WG and Otterson GA: The interaction of histone deacetylase inhibitors and DNA methyltransferase inhibitors in the treatment of human cancer cells. Curr Med Chem Anticancer Agents 3: 187-199, 2003.

41. Vendetti FP and Rudin CM: Epigenetic therapy in non-smallcell lung cancer: Targeting DNA methyltransferases and histone deacetylases. Expert Opin Biol Ther 13: 1273-1285, 2013.

42. Ben Gacem R, Hachana M, Ziadi S, Ben Abdelkarim S, Hidar S and Trimeche M: Clinicopathologic significance of DNA methyltransferase 1, 3a, and $3 \mathrm{~b}$ overexpression in Tunisian breast cancers. Hum Pathol 43: 1731-1738, 2012.

43. Girault I, Tozlu S, Lidereau R and Bièche I: Expression analysis of DNA methyltransferases $1,3 \mathrm{~A}$, and $3 \mathrm{~B}$ in sporadic breast carcinomas. Clin Cancer Res 9: 4415-4422, 2003.

44. Mizuno S, Chijiwa T, Okamura T, Akashi K, Fukumaki Y, Niho Y and Sasaki H: Expression of DNA methyltransferases DNMT1, 3A, and 3B in normal hematopoiesis and in acute and chronic myelogenous leukemia. Blood 97: 1172-1179, 2001.

45. Qu Y, Mu G, Wu Y, Dai X, Zhou F, Xu X, Wang Y and Wei F: Overexpression of DNA methyltransferases $1,3 \mathrm{a}$, and 3b significantly correlates with retinoblastoma tumorigenesis. Am J Clin Pathol 134: 826-834, 2010.

46. Müller BM, Jana L, Kasajima A, Lehmann A, Prinzler J, Budczies J, Winzer KJ, Dietel M, Weichert W and Denkert C: Differential expression of histone deacetylases HDAC1, 2 and 3 in human breast cancer - overexpression of HDAC 2 and HDAC3 is associated with clinicopathological indicators of disease progression. BMC Cancer 13: 215, 2013.
47. Santoro F, Botrugno OA, Dal Zuffo R, et al: A dual role for Hdac1: Oncosuppressor in tumorigenesis, oncogene in tumor maintenance. Blood 121: 3459-3468, 2013.

48. Yang H, Salz T, Zajac-Kaye M, Liao D, Huang S and Qiu Y: Overexpression of histone deacetylases in cancer cells is controlled by interplay of transcription factors and epigenetic modulators. FASEB J 28: 4265-4279, 2014.

49. Fazi F, Travaglini L, Carotti D, et al: Retinoic acid targets DNA-methyltransferases and histone deacetylases during APL blast differentiation in vitro and in vivo. Oncogene 24: 1820-1830, 2005.

50. Shu L, Khor TO, Lee JH, Boyanapalli SS, Huang Y, Wu TY, Saw CL, Cheung KL and Kong AN: Epigenetic CpG demethylation of the promoter and reactivation of the expression of Neurog1 by curcumin in prostate LNCaP cells. AAPS J 13: 606-614, 2011

51. Moseley VR, Morris J, Knackstedt RW and Wargovich MJ: Green tea polyphenol epigallocatechin 3-gallate, contributes to the degradation of DNMT3A and HDAC3 in HCT 116 human colon cancer cells. Anticancer Res 33: 5325-5333, 2013.

52. Fan H, Zhang R, Tesfaye D, Tholen E, Looft C, Hölker M, Schellander K and Cinar MU: Sulforaphane causes a major epigenetic repression of myostatin in porcine satellite cells. Epigenetics 7: 1379-1390, 2012

53. Fang MZ, Wang Y, Ai N, Hou Z, Sun Y, Lu H, Welsh W and Yang CS: Tea polyphenol (-)-epigallocatechin-3-gallate inhibits DNA methyltransferase and reactivates methylation-silenced genes in cancer cell lines. Cancer Res 63: 7563-7570, 2003.

54. Lee WJ, Shim JY and Zhu BT: Mechanisms for the inhibition of DNA methyltransferases by tea catechins and bioflavonoids. Mol Pharmacol 68: 1018-1030, 2005.

55. Kuck D, Caulfield T, Lyko F and Medina-Franco JL: Nanaomycin A selectively inhibits DNMT3B and reactivates silenced tumor suppressor genes in human cancer cells. Mol Cancer Ther 9: 3015-3023, 2010

56. Caulfield $\mathrm{T}$ and Medina-Franco JL: Molecular dynamics simulations of human DNA methyltransferase 3B with selective inhibitor nanaomycin A. J Struct Biol 176: 185-191, 2011.

57. Akhavan-Niaki H and Samadani AA: DNA methylation and cancer development: Molecular mechanism. Cell Biochem Biophys 67: 501-513,2013.

58. Gokul G and Khosla S: DNA methylation and cancer. Subcell Biochem 61: 597-625, 2013.

59. Shu XS, Li L and Tao Q: Chromatin regulators with tumor suppressor properties and their alterations in human cancers. Epigenomics 4: 537-549, 2012.

60. Narayan G, Arias-Pulido H, Koul S, Vargas H, Zhang FF, Villella J, Schneider A, Terry MB, Mansukhani M and Murty VV: Frequent promoter methylation of $C D H 1, D A P K$, $R A R B$, and $H I C 1$ genes in carcinoma of cervix uteri: Its relationship to clinical outcome. Mol Cancer 2: 24, 2003.

61. Kang S, Kim JW, Kang GH, Lee S, Park NH, Song YS, Park SY, Kang SB and Lee HP: Comparison of DNA hypermethylation patterns in different types of uterine cancer: Cervical squamous cell carcinoma, cervical adenocarcinoma and endometrial adenocarcinoma. Int J Cancer 118: 2168-2171, 2006.

62. Braggio E, Maiolino A, Gouveia ME, Magalhães R, Souto Filho JT, Garnica M, Nucci M and Renault IZ: Methylation status of nine tumor suppressor genes in multiple myeloma. Int J Hematol 91: 87-96, 2010.

63. Liu S, Ren S, Howell P, Fodstad O and Riker AI: Identification of novel epigenetically modified genes in human melanoma via promoter methylation gene profiling. Pigment Cell Melanoma Res 21: 545-558, 2008.

64. Xu XL, Yu J, Zhang HY, Sun MH, Gu J, Du X, Shi DR, Wang P, Yang ZH and Zhu JD: Methylation profile of the promoter CpG islands of 31 genes that may contribute to colorectal carcinogenesis. World J Gastroenterol 10: 3441-3454, 2004.

65. Ho AS, Turcan S and Chan TA: Epigenetic therapy: Use of agents targeting deacetylation and methylation in cancer management. Onco Targets Ther 6: 223-232, 2013.

66. Wu CC, Chuang HY, Lin CY, et al: Inhibition of Epstein-Barr virus reactivation in nasopharyngeal carcinoma cells by dietary sulforaphane. Mol Carcinog 52: 946-958, 2013. 